\title{
La falta de servicio ante los daños generados por desastres naturales (Corte Suprema)
}

\author{
Comentario de Gustavo Poblete Espindola*
}

Santiago, veintinueve de abril de dos mil catorce.

\section{Vistos:}

En estos autos rol N 6373-2010 del Segundo Juzgado Civil de Concepción, comparece Luis Antonio Salgado Delgado en representación de su hijo menor, Rodrigo Andrés Salgado Fuentes, deduciendo demanda de indemnización de perjuicios en contra del Fisco de Chile por falta de servicio.

Refiere en su libelo que en la madrugada del día 27 de febrero de 2010, su hijo de 16 años de edad, se encontraba en la comuna de Chiguayante junto a Luis Hernán Soto Repiso y Eduardo Contreras Rodríguez en el domicilio del primero. Ocurrido el terremoto, viajó junto a éstos en el automóvil de uno de ellos en busca de sus familiares que se hallaban en la ciudad de Talcahuano. Señala el demandante que en vista de la información entregada por la radio de que no había alerta de maremoto, aquéllos se dirigieron a Talcahuano por la ruta denominada Interportuaria, donde fueron alcanzados por las olas, permaneciendo Salgado Fuentes conciente y luchando por cerca de treinta minutos en el agua barrosa y sobre los escombros que ésta arrastraba. Fue rescatado por un grupo de personas que escucharon sus gritos de auxilio, sufriendo múltiples heridas y contusiones. Este episodio, agrega, dejó en su hijo un severo trauma que debe actualmente ser tratado por especialistas.

Los actores atribuyen este infortunio al actuar negligente, irresponsable y temerario de la autoridad al entregar información errónea respecto de la no ocurrencia de un maremoto, haciendo creer a la población que no existía riesgo.

Por sentencia de cinco de marzo de dos mil doce, el tribunal de primera instancia acogió la demanda solo en cuanto condenó al Fisco de Chile a pagar a la parte demandante

* Licenciado en Ciencias Jurídicas y Sociales, Universidad Alberto Hurtado. Profesor Instructor de Derecho Constitucional de la Universidad Alberto Hurtado. Ayudante de investigación del proyecto FONDECYT N 1140982 sobre "Análisis diacrónico de la influencia de las labores dogmáticas en las tareas judiciales: el caso de la nulidad de derecho público (1990-2005)” (Programa 2014-2015). Correo electrónico: gustavo.poblete.espindola@gmail.com. 
la suma total de $\$ 500.000$ por concepto de daño moral. Se dictaminó que el perjuicio cuya indemnización se reclamaba tenía dos causas: en primer lugar, la culpa de la víctima al ingresar a Talcahuano por la ruta Interportuaria después de un terremoto de inusual magnitud que causó grandes daños materiales de los que debió haberse percatado, como las diversas grietas y levantamiento en las restantes vías de ingreso a Talcahuano, las que se hallaban igualmente transitables adoptando las precauciones del caso, pero no obstante ello, decidió ingresar a ese puerto por una zona evidente de inundación. Y en segundo lugar, la culpa del demandado al proporcionar una información equivocada a través de sus órganos acerca de la inexistencia de un maremoto, el cual ya se había iniciado con la llegada de la primera ola a las costas de la región veinte minutos después del sismo principal, deficiencia, según estima el sentenciador de primer grado, que constituye falta de servicio de los órganos de la Administración del Estado, y que llevó a la víctima a dirigirse desde su domicilio en Chiguayante hacia Talcahuano por la ruta Interportuaria, donde fue alcanzado por una ola (considerando vigésimo cuarto de la sentencia de primera instancia).

Apelado ese fallo por el demandado, la Corte de Apelaciones de Concepción mediante sentencia de cinco de noviembre de dos mil trece lo revocó, desestimando la demanda. Razonó que del mérito de la prueba testimonial producida por los demandantes se advierte su insuficiencia para dar por acreditado en autos que la víctima decidió emprender viaje en un vehículo desde Chiguayante a Talcahuano con pleno conocimiento que la autoridad pública había informado a la población que no había alerta ni ocurriría un maremoto, o si por el contrario, resolvió acometer dicho viaje sin saber realmente de esa información, toda vez que los testimonios aportados por los demandantes serían absolutamente genéricos en ese punto y, por tanto, no permitían a la Corte formarse convicción de que el occiso conociera personalmente de ese hecho, sea por haberlo oído de Carabineros o Bomberos, o al haberlo escuchado a través de una transmisión radiofónica (considerando cuarto de la sentencia de segunda instancia).

Añaden los jueces del tribunal de alzada que, por consiguiente, el hecho en que se funda la falta de servicio que se imputa a la Administración no tiene relación de causalidad con el hecho dañoso (considerando quinto del mismo fallo).

Por último, citando una sentencia de la Corte Suprema recaída sobre este mismo hecho (Rol No 1250-2012, iniciado por el padre de Luis Soto Repiso), se señaló: "Sin entrar a pronunciarse sobre si la conducta constituye o no falta de servicio, lo cierto es que al no poder vincular la actuación cuestionada y reprochada por el actor, a la salida que hiciera la víctima hacia Talcahuano por la ruta Interportuaria y el posterior desenlace fatal, la demanda no puede prosperar".

En contra de esta última determinación, la parte demandante interpuso recurso de casación en el fondo.

Se trajeron los autos en relación.

\section{Considerando:}

Primero: Que en el recurso de casación se denuncia, en primer término, la infracción de los artículos 4 y 42 de la Ley No 18.575 sobre Bases de la Administración del Estado, 
puesto que la sentencia impugnada eximió de responsabilidad al Estado pese a la irrefutable y colosal falta de servicio en que incurrió con motivo del actuar de la primera autoridad política regional, el Intendente de la época, quien en la madrugada del 27 de febrero de 2010 aseguró a la población la inexistencia de un tsunami con ocasión del terremoto habido a las 3:34 horas. Manifiestan los recurrentes que fue a consecuencia del decir público del Intendente de la época, Jaime Tohá, que Rodrigo Salgado Fuentes se expuso al daño sufrido en las aguas de mar que inundaron la vía Interportuaria.

Añade que dadas las condiciones en que se encontraba el país luego del terremoto, esto es, colapsado el sistema de telecomunicaciones, paralizado el gobierno y el control de la Administración, la autoridad estaba imposibilitada de tener un conocimiento completo de cuánto ocurría y, por ende, resultaba elemental mantener vigente toda medida de cuidado y prevención. Así, era claro que no era posible descartar el riesgo cierto de sobrevenir un tsunami como el que a la postre se produjo.

Menciona que el propio demandado ha indicado en su defensa una serie de circunstancias de anormalidad para justificar el error en la información; por lo mismo, tales condiciones aconsejaban no realizar un llamado como el que hizo el Intendente de ausencia de riesgo, toda vez que la autoridad no contaba efectivamente con antecedentes ciertos para minimizar un riesgo de tsunami. Expresa que el obrar temerario de la autoridad constituye la falta de servicio de que nace la responsabilidad del Estado y su obligación de indemnizar.

Segundo: Que, a continuación, el recurrente acusa la vulneración del artículo 1698 del Código Civil, puesto que los demandantes estaban relevados de acreditar la falta de servicio desde que su constatación se revela de la propia defensa del demandado en cuanto a la forma en que actuó la autoridad. Precisa, sin embargo, que aun en el caso de que la carga de la prueba hubiere correspondido a los demandantes, estima improcedente reducir el quid de la cuestión a poner en tela de juicio que la víctima haya escuchado la aludida información antes de emprender viaje. Entenderlo así, sostiene, importa garantizar impunidad legal en todo evento al error administrativo.

Expone que también yerra la sentencia al no estimar como hecho público y notorio el que todos quienes se encontraban en la zona de Concepción en la madrugada del 27 de febrero de 2010 escucharon el llamado de la autoridad a volver a sus casas puesto que no había alerta de maremoto, con lo cual se conculca lo dispuesto en el artículo 89 del Código de Procedimiento Civil, ya que se obvia un hecho y sus consecuencias procesales.

Finalmente argumenta que al desecharse las presunciones emanadas de las pruebas testimoniales a fin de tener por acreditado que se escuchó a la autoridad, se transgrede el artículo 383 del Código de Procedimiento Civil.

Tercero: Que en lo que interesa al recurso, son hechos no discutidos en la causa los siguientes:

a) Que el Intendente de la Octava Región concedió dos entrevistas a radio Bío Bío, a las 5.01 AM y 5.19 AM del día 27 de febrero de 2010 transmitidas a la comunidad, relativa a la inexistencia de riesgo de maremoto (Oficio de Radio Bío Bío de fojas 332). 
b) Que ocurrido el terremoto a las 3:34 horas del 27 de febrero de 2010, Rodrigo Salgado Fuentes junto con Luis Soto Repiso y Eduardo Contreras Rodríguez salieron desde Chiguayante hacia la ciudad de Talcahuano a través de la ruta Interportuaria, siendo alcanzados por las olas producto del tsunami que sucedió al terremoto.

c) Que la primera ola del maremoto que afectó a Talcahuano se produjo a las 3:54 horas del día 27 de febrero de 2010.

Cuarto: Que los jueces de segunda instancia estimaron necesario acreditar que la víctima hubiera escuchado la información emitida por el señor Intendente a través de radio Bío Bío antes de emprender el viaje. Ello, por cuanto en la demanda se sostiene que fue la información errónea comunicada por el Intendente de que no existía riesgo de maremoto la causa que llevó a la víctima a viajar desde Chiguayante con destino a Talcahuano por la ruta Interportuaria, donde fue alcanzado por una ola de mar producto del maremoto.

Conforme a dicho razonamiento analizaron la prueba testimonial rendida por el actor, conformada por las declaraciones de dos testigos de oídas, juzgando que resultaba insuficiente para dar por acreditado que Rodrigo Salgado Fuentes había decidido emprender viaje en vehículo desde Chiguayante hacia Talcahuano impulsado por la comunicación pública del Intendente de la época y que fuera recogida por otros organismos, como Carabineros y Bomberos, o bien, si por su propia determinación y desconociendo la aludida información, viajó hacia Talcahuano en busca de sus familiares domiciliados en esa ciudad.

De allí concluyeron los magistrados que no era posible vincular la actuación de la autoridad tildada de falta de servicio con la decisión de la víctima de emprender viaje hacia Talcahuano por una ruta que bordea la costa momentos después de sucedido un sismo de extraordinaria magnitud.

Quinto: Que la falta de servicio ha sido definida doctrinaria y jurisprudencialmente como aquella en que el servicio actúa mal, lo hace tardíamente o no actúa, vale decir es el fundamento jurídico en cuya virtud los costos de los daños sufridos por un particular son asumidos por la Administración, conforme disponen los artículos 42 de la Ley $\mathrm{N}^{\circ}$ 18.575, Orgánica Constitucional de Bases Generales de la Administración del Estado, 152 de la Ley No 18.695, Orgánica Constitucional de Municipalidades y 38 de la Ley $N^{\circ} 19.966$ sobre Garantías Explícitas en Salud.

La falta de servicio es considerada como "la culpa del Servicio", y en consecuencia deberá probarse -por quien la alega- el mal funcionamiento del servicio, el funcionamiento tardío o el no funcionamiento del mismo; que esta omisión o acción defectuosa haya provocado un daño al usuario o beneficiario del servicio público de que se trata; $y$, en fin, que la falla en la actividad del ente administrativo haya sido la causa del daño experimentado, todo por disponerlo así el artículo 42 de la Ley de Bases de la Administración del Estado.

Dentro de este contexto el actor imputó al Estado una falla en su actuación, cual era informar por medios radiales a través del señor Intendente de la Región del Bío Bío 
que no existía riesgo de tsunami después del terremoto acaecido el día 27 de febrero de 2010, información que, sabido es, resultó errada pues el fenómeno natural se produjo con lamentables consecuencias.

Sin embargo, más allá del objetivo error en la información y la eventual calificación de la misma, resulta certera la exigencia que han requerido los jueces del fondo en orden a la necesidad de demostrar que la víctima, como consecuencia de esa información, decidió salir desde Chiguayante a Talcahuano por la ruta Interportuaria, y ello es así porque en dicha circunstancia descansa precisamente el fundamento de la demanda, reiterado además en el escrito de casación cuando se dice a fojas 405: "La cuestión basal de que trata la presente causa, es la exigencia de responsabilidad de la administración del Estado, por omisión de servicio, que particularmente se acota, por el obrar de la primera autoridad politica regional, que en la madrugada del 27 de febrero de 2010, aseguró a la población la inexistencia de un maremoto a consecuencia del terremoto habido a las 03,34 hrs. Pues bien, a consecuencia del decir público del Sr. Intendente de la época don Jaime Tohá, don Rodrigo Andrés Salgado Fuentes, se expuso al daño sufrido en las aguas de mar, que inundaron la vía Interportuaria".

Lo expuesto muestra que ha sido el propio actor quien ha ligado la salida de su hijo hacia Talcahuano con la información entregada por la autoridad. Es por ello entonces que corresponde que quien afirma dicho aserto lo demuestre, siguiendo la clara regla probatoria instituida en el artículo 1698 del Código Civil, de lo que se sigue que debe descartarse una vulneración a este precepto porque se ha dado en la sentencia una correcta aplicación a dicha norma. En efecto, este último precepto prescribe en su inciso primero que incumbe probar las obligaciones o su extinción al que alega aquéllas o ésta, de modo que su infracción se configura en la medida que el fallo altere el peso de la prueba, pues esta disposición impone imperativamente esta carga, como regla general, a quien alega la existencia de la obligación o su extinción.

Luego, para efectos de conformar o no los fundamentos de la demanda a los presupuestos de la acción impetrada, la sentencia cuestionada les exigió justificar los requisitos que hacen surgir la responsabilidad reclamada, entre ellos, la relación causal entre el hecho que se dice ilícito y los daños alegados, ineludible en toda indemnización de perjuicios por responsabilidad extracontractual. Por lo mismo, al asignar así el peso probatorio, es evidente que el fallo no ha incurrido en infracción alguna.

Más bien, de los argumentos expuestos por el recurrente se desprende que lo reprochado a la sentencia es haber concluido que no se demostró dicho supuesto de la acción, aseverando que tal carga fue satisfecha con la prueba testimonial rendida. Tal pretendido yerro no dice relación con la vulneración del artículo 1698 citado, sino que a una cuestión que le resulta ajena, puesto que se refiere a la apreciación de un medio probatorio específico.

Sexto: Que, en consecuencia, al haberse resuelto la ausencia de vínculo de causalidad entre la eventual falta de servicio y los daños alegados no se advierte error de derecho al decidir de ese modo, puesto que no se demostró que exista una correspondencia de causa a efecto entre la información proporcionada por el Intendente en cuanto descartar el riesgo de tsunami y el resultado denunciado. 
Séptimo: Que, por otra parte, el recurso denuncia la vulneración referida a la prueba del hecho notorio, afirmando que todos en Concepción y sus alrededores escucharon la madrugada del 27 de febrero de 2010 el llamado de la autoridad de volver a las casas porque no había alerta de maremoto. Sin embargo, tal hecho no fue establecido por la sentencia en alzada, de manera que tampoco existe violación a la norma del artículo 89 del Código de Procedimiento Civil, porque el supuesto fáctico en el que descansa la afirmación no es un hecho de la causa que haya sido consignado por los sentenciadores del grado.

Octavo: Que en cuanto a la valoración que los sentenciadores hicieron de los testimonios presentados en la causa y su eventual calificación como base de presunción judicial en los términos del artículo 383 del Código de Procedimiento Civil, de la sola lectura de este precepto se desprende que no reviste el carácter de norma rectora de la prueba, toda vez que dispone que los jueces del mérito tienen facultades soberanas y privativas para ponderar las declaraciones de los testigos de oídas como base de una presunción judicial, atribuciones que por sus propias características no pueden estar sujetas a la revisión de un tribunal de casación.

Noveno: Que al rechazarse los distintos yerros de derecho planteados por los demandantes en su recurso de casación en el fondo, corresponde que este sea desestimado. Efectivamente, como antes se advirtió, esta Corte Suprema al conocer de un recurso de casación en el fondo está impedida de revisar la forma en que se establecieron los hechos por los jueces de la instancia, sin embargo, en el evento que éstos incurran en errores en la aplicación de las denominadas leyes reguladoras de la prueba, de manera excepcional es posible la revisión de esa labor por una Corte de casación. Pero solo en el evento de infracción a tales disposiciones, pues existen otras que estando relacionadas con la prueba, son de soberanía específica de los jueces de fondo, tales como las que fueron invocadas por los recurrentes.

Por consiguiente, no habiéndose probado que la víctima escuchó la comunicación de la autoridad, no es posible determinar jurídicamente si hubo relación de causalidad entre ese hecho con haber sido alcanzado por el maremoto que azotó a la región.

Por estas consideraciones y lo dispuesto en los artículos 764, 765, 767 y 805 del Código de Procedimiento Civil, se rechaza el recurso de casación en el fondo deducido en lo principal de fojas 402 en contra de la sentencia de cinco de noviembre de dos mil trece, escrita a fojas 399.

Acordada con el voto en contra del Ministro señor Aránguiz, quien fue de parecer de acoger el recurso de casación en el fondo y consecuentemente dar lugar a la demanda indemnizatoria en virtud de las siguientes consideraciones:

$1^{\circ}$. Que el contenido del mensaje del Intendente afirmando la inexistencia de riesgo de maremoto y llamando a mantenerse en las casas configura un deficiente obrar del ente estatal, puesto que la ausencia de informaciones, las deficiencias técnicas, la falta de comunicaciones y, en general, las circunstancias excepcionales que 
se vivían en el país a consecuencia del devastador terremoto, no permitían tan categórica instrucción de parte de la autoridad. Ello, asimismo, provocó que las autoridades tampoco adoptaran inmediatamente medidas de resguardo esenciales para proteger a la población, como la pronta evacuación de las zonas inundables de la región, característica que revestía la ruta que eligió la víctima para trasladarse desde su domicilio en Chiguayante a la ciudad de Talcahuano. Hubo un Estado ausente, pues aun considerando las circunstancias extraordinarias que afectaban a gran parte del territorio nacional, su comportamiento estuvo lejos de ser aquel que era posible exigirle.

$2^{\circ}$. Que en lo concerniente a la prueba de la relación causal, el demandante expresó que su hijo actuó motivado por las informaciones vertidas no solo por el Intendente, sino también por Carabineros y Bomberos, quienes probablemente haciendo eco de las palabras del primero en una entrevista radial, aseguraban a la población que no existía peligro de tsunami, por lo que no instruían a las personas a dirigirse a lugares alejados del mar. Es del parecer de quien disiente que extremar la carga probatoria en circunstancias tan anormales como las que sucedieron a esta catástrofe, exigiendo evidencias fehacientes y prolijas de que el afectado luego de escuchar de las mencionadas autoridades la ausencia de riesgo decidió emprender un viaje bordeando la costa, deviene en imponer al demandante una tarea prácticamente imposible de satisfacer.

$3^{\circ}$. Que de los antecedentes de hecho no controvertidos en esta causa, esto es, terremoto, entrevista radial del Intendente, viaje de Rodrigo Salgado Fuentes desde Chiguayante a Talcahuano y tsunami, se hace razonablemente plausible el planteamiento del demandante de que la víctima actuó con conocimiento de que las autoridades habían descartado cualquier riesgo de tsunami, pudiendo incluso desplazarse por una vía que enfrentaba la playa cuyo acceso no había sido restringido. De allí que en concepto de este disidente, se está en condiciones de establecer la relación de causalidad, por tratarse de un aspecto de Derecho sustentado en los hechos acreditados.

$4^{\circ}$ Que vulnerado ha sido de esta manera el artículo 1698 del Código Civil que contiene la pauta básica en materia de carga de la prueba, pues los jueces de la instancia han desconocido la existencia de antecedentes suficientemente ilustrativos de los hechos que fundan la acción intentada.

Regístrese y devuélvase con sus agregados.

Redacción a cargo del Abogado Integrante señor Prieto y de la disidencia, su autor. Rol No 16.920-2013.

Pronunciado por la Tercera Sala de esta Corte Suprema integrada por los Ministros Sr. Rubén Ballesteros C., Sra. Rosa Egnem S., Sr. Carlos Aránguiz Z., Sra. Andrea Muñoz S., y el Abogado Integrante Sr. Alfredo Prieto B. No firma, no obstante haber concurrido a la vista y al acuerdo de la causa, el Abogado Integrante señor Prieto por estar ausente. Santiago, 29 de abril de 2014. 
Autoriza la Ministra de Fe de la Excma. Corte Suprema.

En Santiago, a veintinueve de abril de dos mil catorce, notifiqué en Secretaría por el Estado Diario la resolución precedente.

\section{COMENTARIO}

En nuestro ordenamiento jurídico resulta complejo realizar una correcta asociación entre las obligaciones del Estado y su correspondiente responsabilidad en aquellos casos de incumplimiento de sus funciones. Establecer esta relación de causalidad se torna aún más dificultosa en el momento que se pretende determinar una reparación de los daños ocasionados en situaciones de catástrofes naturales.

De la lectura del fallo transcrito se desprende que la Corte Suprema rechaza el recurso de casación en el fondo deducido, por no haberse probado que la víctima escuchó la comunicación de la autoridad acerca de la inexistencia de un maremoto, es decir, que no es posible determinar jurídicamente si hubo relación de causalidad entre ese hecho con haber sido alcanzado por el maremoto que azotó a la región.

En este contexto excepcional, el estándar de comportamiento exigible al órgano de la Administración es un elemento necesario para juzgar su responsabilidad por las omisiones incurridas en la fase de prevención del maremoto. Desde esta perspectiva, la responsabilidad del Estado frente a los desastres naturales será siempre limitada, es por ello que la razonabilidad de las decisiones adoptadas por los jueces debe contemplar las condiciones de emergencia en que esas medidas se adoptan ${ }^{1}$.

La manera más utilizada de manifestar esta limitación estatal es la que aprecia el desastre natural como un evento de fuerza mayor ${ }^{2}$. Si se considera el terremoto y consiguiente maremoto como un evento de fuerza mayor puede tener una doble consideración, ya que puede excluir la relación de causalidad o bien puede incidir en la falta de servicio mediante la alteración de las exigencias normales que resultan esperables de un servicio público. De esta manera, el impacto dañoso de un desastre natural se enfrenta a una organización administrativa cuya envergadura económica dependerá de la forma en que la decisión legal democrática haya resuelto la inversión presupuestaria, para reparar los perjuicios generados en este tipo de situaciones ${ }^{3}$.

En el mismo sentido, se puede afirmar que ante el acontecimiento de un desastre natural, como el acontecido el 27 de febrero de 2010, se afecta gravemente el normal desenvolvimiento de las instituciones del Estado, porque, por un lado, estamos en presencia de una situación en que sobrevienen destrucciones y daños materiales que amenazan

${ }^{1}$ Lerner, K. (1991), “Governmental Negligence Liability Exposure in Disaster Management”, en The Urban Lawyer, vol. 3, $\mathrm{N}^{\circ}$ 3, p. 352.

${ }^{2}$ El artículo 45 del Código Civil, define la fuerza mayor como “... el imprevisto a que no es posible resistir, como un naufragio, un terremoto...".

${ }^{3}$ Letelier, R. (2012), "Falta de servicio en situaciones de catástrofes naturales", en Letelier, R. (Coord.), La falta de servicio, Thomson Reuters, Santiago, Chile, p. 311. 
la vida y la salud de las personas y, por otro lado, la intervención de los órganos de la Administración no es armónico con la magnitud del acontecimiento natural.

Así, se puede concluir que el criterio utilizado por la Corte Suprema para determinar la inexistencia de la relación causal en el caso concreto, descartando la culpa del Servicio, es en atención a la situación de anormalidad que genera una catástrofe natural de gran escala, y a su vez, la dificultad que se produce para probar la falta de servicio de la Administración, ya que estos desastres naturales son muy complejos de manejar y controlar, principalmente en las primeras horas de ocurrido el hecho. 